\title{
Plastic deformation of metallic glasses: Size of shear transformation zones from molecular dynamics simulations
}

\author{
Mareike Zink, ${ }^{1}$ K. Samwer, ${ }^{1}$ W. L. Johnson, ${ }^{2}$ and S. G. Mayr ${ }^{1, *}$ \\ ${ }^{1}$ I. Physikalisches Institut, Georg-August-Universität Göttingen, Friedrich-Hund-Platz 1, 37077 Göttingen, Germany \\ ${ }^{2}$ 138-78 Keck Laboratory of Engineering, California Institute of Technology, Pasadena, California 91125, USA
}

(Received 19 April 2006; published 24 May 2006)

\begin{abstract}
Plastic deformation in metallic glasses well below their glass transition temperatures $T_{g}$ occurs spatially heterogeneously within highly localized regions, termed shear transformation zones (STZs). Yet, their size and the number of atoms involved in a local shear event, remains greatly unclear. With the help of classical molecular dynamics (MD) computer simulations on plastic deformation of the model glass CuTi during pure shearing, we address this issue by evaluating correlations in atomic-scale plastic displacements, viz. the displacement correlation function. From the correlation length, a universal diameter of about $15 \AA$, or, equivalently, approximately 120 atoms is derived for a variety of conditions, such as variable strains, strain rates, temperatures, and boundary conditions. Our findings are consistent with a recent model proposed by Johnson and Samwer [Phys. Rev. Lett. 95, 195501 (2005)].
\end{abstract}

DOI: 10.1103/PhysRevB.73.172203

PACS number(s): 61.43.Fs, 62.20.Fe, 02.70.Ns

The physics of plastic deformation and flow in glassesone of the oldest engineering materials of mankind-is still poorly understood when compared to their crystalline counterparts, and thus has attracted increasing experimental and computational interests during the past decades. ${ }^{1-5}$ In particular the highly heterogeneous, cooperative dynamics on multiple time and length scales, ${ }^{6}$ and its influence on macroscopic dimensions is still greatly unclear, presumably due to the lack of an atomic-scale picture. This includes the impact on important material properties, such as elastic modulus, fracture behavior, and plastic flow. Adam and Gibbs ${ }^{7}$ were the first to invoke the concept of a cooperative rearranging region (CRR) back in 1965 by proposing clusters that reorganize during shearing. As a main weakness of their treatment, however, they fail to predict a specific cluster size. Subsequent ideas developed by Cohen et al., ${ }^{8}$ Spaepen, ${ }^{9}$ Argon, ${ }^{10,11}$ and others describe flow and creep in metallic glasses as activated transformations in intrinsic dynamic heterogeneities, termed shear transformation zones (STZs). ${ }^{12}$ These models characterize plastic flow in metallic glasses during shearing as a local event appearing as a spontaneous and cooperative reorganization of individual clusters (STZs). ${ }^{13-16}$ As a major problem, however, the size of STZs still remains unclear, in particular for realistic material systems. A first hint to remedy this deficiency was provided by Debenedetti and Stillinger ${ }^{17}$ who introduced a potential energy landscape model for the amorphous state and yielding in glasses. Johnson and Samwer ${ }^{18}$ extended their treatment and proposed a size of STZs of the order of 100 atoms from energetic considerations.

In the present investigations we employ molecular dynamics (MD) computer simulations to corroborate these predictions and link atomic-scale kinetics and mechanical properties across these length scales by determining the size of STZs as a function of macroscopic quantities. This is accomplished by investigating shear events below $T_{g}$ for the model glass CuTi. Evaluations of the plastic contribution to atomicscale displacements facilitate to identify cooperative motions, as expected in dynamical heterogeneities or during shear banding. The size of STZs is determined by calculating a correlation function for plastic displacements (displacement correlation function-DCF) which offers the possibility to systematically access the diameter of these zones directly from MD data.

MD simulations are performed using a highly realistic interatomic potential of the embedded-atom type (EAM) ${ }^{19}$ which has been parametrized for CuTi by Sabochick and Lam. ${ }^{20}$ In particular the mechanical, structural, and thermal properties of the crystalline and amorphous phase compare well with experiments, as indicated in previous studies. ${ }^{21-23}$ The control of temperature and pressure to achieve isothermal and isobaric conditions is obtained by a Berendsen ${ }^{24}$ thermostat and barostat, respectively. The CuTi simulation cell consists of 35,152 atoms, arranged in a cubic shape with a side length $L \approx 80 \AA$, and periodic boundary conditions with zero pressure applied in all directions. Relaxed amorphous cells are obtained by quenching the liquid phase from 4500 down to $10 \mathrm{~K}$, while we chose the quench rate through the glass transition low enough $(0.1 \mathrm{~K} / \mathrm{ps})$ to allow for sufficient relaxation. This is also inferred from successive heating and quenching cycles as well as the time dependence of the mean square displacements. ${ }^{21,23}$ The change of volume and enthalpy as a function of temperature during quenching indicates a glass transition temperature $T_{g}^{q u e n c h}=610 \mathrm{~K}$. Besides monitoring the signatures of the glass transition in volume and enthalpy, the amorphous nature of the cell is confirmed by calculating the radial distribution function. Subsequent heating to $1500 \mathrm{~K}$ with an increased rate $(1 \mathrm{~K} / \mathrm{ps})$ yields $T_{g}^{\text {heat }}=850 \mathrm{~K} .{ }^{23}$ We employ this heating rate also to reach the base temperature $(500 \mathrm{~K})$ of the simulation experiments on STZs.

After reaching the base temperature, two cells with different types of boundary conditions are prepared: For the first cell, only the periodic boundaries in $z$ directions are released, while the topmost $(+z$ direction $)$ and bottommost $(-z$ direction) four atomic layers are fixed and the cell dimensions in $x$ and $y$ directions are kept constant. To investigate the impact of the absence of periodic boundary constraints, a sec- 


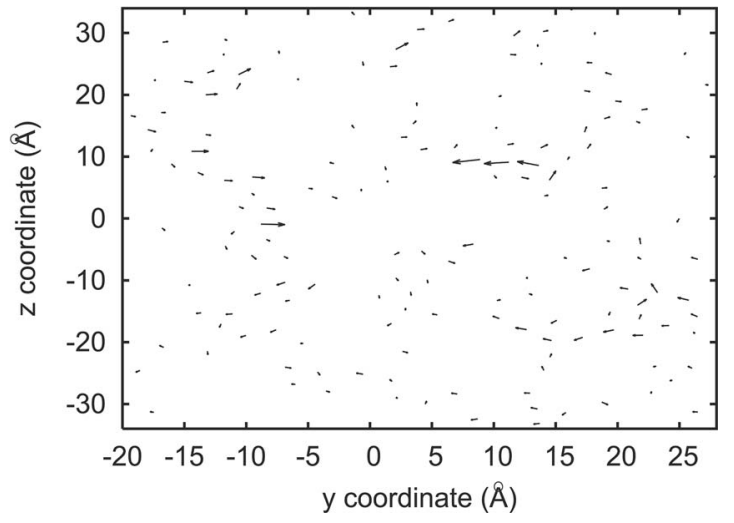

FIG. 1. Slice of the plastic displacement plot during shearing of a CuTi cell with periodic boundaries at $500 \mathrm{~K}$ and $6 \%$ strain, $\dot{\gamma}=1 /$ ns. Only atoms displaced more than $0.45 \AA$ are shown.

ond cell with open boundaries also in $x$ and $y$ directions is prepared. Both cells are additionally relaxed for at least $10 \mathrm{~ns}$ after opening the boundaries to relax the open surfaces.

By moving the topmost four atomic layers in the $+y$ direction with constant velocity, three predefined strain rates, $\dot{\gamma}_{1}=0.5 \times 10^{9} \mathrm{~s}^{-1}, \quad \dot{\gamma}_{2}=1.0 \times 10^{9} \mathrm{~s}^{-1}$, and $\dot{\gamma}_{3}=10.0 \times 10^{9} \mathrm{~s}^{-1}$ are imposed on the cells, while the corresponding shear stresses are extracted from the virials. In the following we focus on the strain levels of individual atoms below or near the yielding point of the stress-strain curves. To investigate the plastic contribution $\vec{d}_{i}$ to the total displacement of an individual atom $i$, elastic contributions are eliminated by transforming the cell back to its original, viz. unsheared, shape by an appropriate linear regression. Figure 1 shows an exemplary $2 \AA$ thick slice of the plastic displacement field of all atoms at $500 \mathrm{~K}, 6.0 \%$ strain (right below the yielding point, $6.5 \%)$ and a strain rate of $1.0 \times 10^{9} \mathrm{~s}^{-1}$. To eliminate thermal fluctuations, only atoms with displacements $d_{i}=\left|\vec{d}_{i}\right|>0.45 \AA$ are drawn. Figure 1 clearly indicates the presence of mobile clusters of the order of $10-15 \AA$ within a rigid glass matrix, which are interconnected via single strings of mobile atoms. Both clusters and interconnections together seem to form a dynamic network of plastic deformation, which controls the macroscopic behavior. It is noteworthy, however, that-unlike in shear bands - the atoms within the clusters do not have a preferred moving direction and displace rather randomly.

Comparable networks of clusters and interconnects are observed for any of the investigated strains, strain rates, and temperatures $(10 \mathrm{~K}$ and $500 \mathrm{~K})$ below the corresponding yielding points. Only the number of clusters and the distance that atoms move plastically seems to vary, e.g., increases for higher strains or temperatures. For open boundaries we generally find that the yielding point is located at higher strains and lower stresses than for periodic boundary conditions. This is due to the fact that shear bands can easily nucleate at all open surfaces, and subsequently percolate and permeate allover the cell. In the case of periodic boundaries, our studies indicate that shear bands build up locally and do not percolate before the yielding point is reached. However, the presence and nature of the STZs again is greatly independent

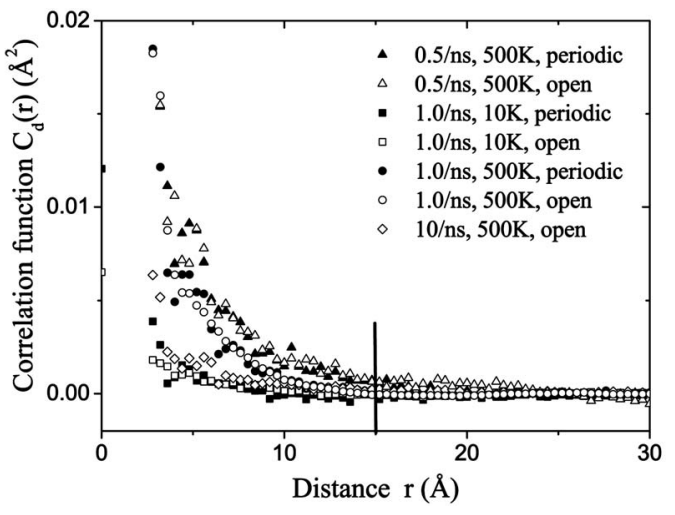

FIG. 2. DCF at $4.0 \%$ strain, as determined for different strain rates, temperatures and boundary conditions (open and filled symbols for open and periodic boundaries, respectively). The cutoff to eliminate thermal vibrations was chosen as 0.4 and $0.2 \AA$ for 500 and $10 \mathrm{~K}$, respectively.

of the type of chosen boundaries below the yielding point.

To evaluate the apparent (Fig. 1) size of the STZs quantitatively, we choose to employ a spatial correlation function $C_{d}(r), r=|\vec{r}|$, of the absolute plastic displacement field $d(\vec{r})=|\vec{d}(\vec{r})|$, which can be regarded as an extension of the individual atomic plastic displacements $d_{i}=\left|\vec{d}_{i}\right|$, to the continuum limit, viz. $d\left(\vec{r}_{i}\right)=d_{i}$,

$$
\begin{aligned}
& C_{d}(r)=\langle d(\vec{R}+\vec{r}) \cdot d(\vec{R})\rangle_{\vec{R} ;|\vec{r}|=r} \\
& -\langle d(\vec{R}+\vec{r})\rangle_{\vec{R} ;|\vec{r}|=r} \cdot\langle d(\vec{R})\rangle_{R} ;|\vec{r}|=r \\
& =\frac{\sum_{i \leqslant j} d_{i} \cdot d_{j} \cdot \delta\left(r-r_{i j}\right)}{\sum_{i \leqslant j} \delta\left(r-r_{i j}\right)} \\
& -\frac{\left(\sum_{i \leqslant j} d_{i} \cdot \delta\left(r-r_{i j}\right)\right) \cdot\left(\sum_{i \leqslant j} d_{j} \cdot \delta\left(r-r_{i j}\right)\right)}{\left(\sum_{i \leqslant j} \delta\left(r-r_{i j}\right)\right)^{2}} \\
& \text { for } \sum_{i \leqslant j} \delta\left(r-r_{i j}\right) \neq 0 .
\end{aligned}
$$

In Eq. (1) \langle\rangle$_{\vec{R} ;|\vec{r}|=r}$ denotes the average of all $\vec{R}$ and successively of all vectors $\vec{r}$ with equal $r$, while Eq. (2) sums over all pair of atoms with $\vec{r}_{i j}=\vec{r}_{i}-\vec{r}_{j}$ and $r_{i j}=\left|\vec{r}_{i j}\right|$. The latter is undefined, in case the denominator vanishes, which-in its discretized version-only occurs for separations $r$ smaller than the next-neighbor distance in amorphous systems. To eliminate thermal vibrations, all displacements below a specified cutoff were omitted, and all atoms closer than $12.5 \AA$ to the open surfaces were not included. Systematic tests on an appropriate choice of the cutoff assured that it did not affect any of the results and conclusions below within reasonable boundaries. As shown in Figs. 2 and 3, the DCF reflects an elevated correlation for cluster diameters of 


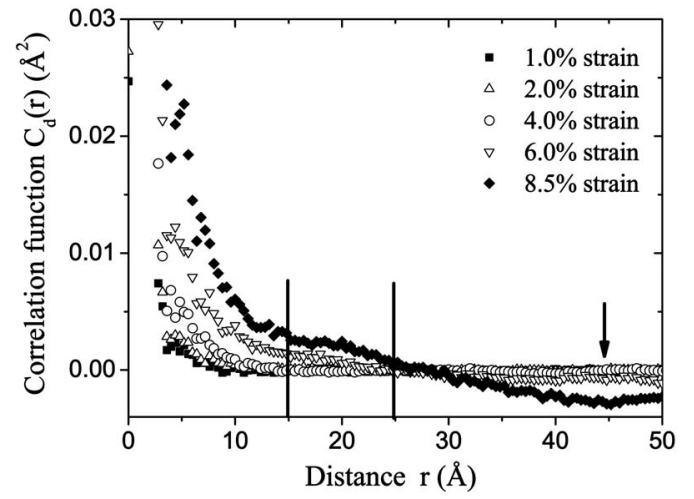

FIG. 3. DCF for variable strain at $500 \mathrm{~K}$, open boundaries, strain rate $1.0 / \mathrm{ns}$, cutoff $0.4 \AA$.

$\approx 15 \AA$ and below, indicating a cooperative motion on these length scales, which can be identified as dynamic heterogeneities or, equivalently, STZs. Within the scope of our investigations on $\mathrm{CuTi}$, this number seems universal without any dependencies on strain, strain rates, temperature (below $T_{g}$ ), and boundary conditions. The occurrence of even higher correlations in the DCF might be inhibited by the presence of thermal noise, as well as thermal and shear induced mobility among neighboring atoms. We associate the plateau in the DCF, as seen in Fig. 3 between $r=15 \AA$ and $r=25 \AA$, with sequences of atomic exchange processes, connecting the highly mobile clusterlike regions. For strains close to the yielding point and beyond, the shape of the DCF also reflects the presence of shear bands, as indicated by the arrow in Fig. 3 , where a shear band penetrates the cell in the $y$ direction. For higher temperatures and strains the overall level of the correlation function increases, as expected from Eq. (1).

To conclude, our MD simulations indicate that macroscopic shear deformation of amorphous $\mathrm{CuTi}$, and presumably also other metallic glasses, under a variety of conditions is mediated by shear transformation zones of $\approx 15 \AA$ in diameter, which are interconnected by a dynamical stringlike displacement network. The observation of STZs of approximately 120 atoms is in good agreement with a recent model proposed by Johnson and Samwer ${ }^{18}$ that postulates an energetically favorable size of STZs of the order of 100 atoms. A comparison with the works of Schober, ${ }^{25,26}$ who detected stringlike behavior for unsheared amorphous materials close to the glass transition temperature raises the question if STZs are formed at the crossings of the strings, or if strings are the transport agents between individual STZs. Also with respect to this question work is left for the future.

\section{ACKNOWLEDGMENTS}

M. Zink wishes to acknowledge the support by M. Jungsbluth and T. Edler for their help with the script programming for data analysis. Financial support by the German DFGSFB 602 and the G.I.F, Grant No. G-2103-1428.14/2004 as well as a grant of computing time by the Gesellschaft für wissenschaftliche Datenverarbeitung Göttingen (GWDG) is gratefully acknowledged.
*Author to whom correspondence should be addressed. Email address: smayr@uni-goettingen.de

${ }^{1}$ D. Srolovitz, V. Vitek, and T. Egami, Acta Metall. 31, 335 (1983).

${ }^{2}$ S. Kobayashi, K. Maeda, and S. Takeuchi, Acta Metall. 28, 1641 (1980).

${ }^{3}$ J. Lu, G. Ravichandran, and W. L. Johnson, Acta Mater. 51, 3429 (2003).

${ }^{4}$ C. D. Lorenz and M. J. Stevens, Phys. Rev. E 68, 021802 (2003).

${ }^{5}$ F. Varnik, L. Bocquet, J.-L. Barrat, and L. Berthier, Phys. Rev. Lett. 90, 095702 (2003).

${ }^{6}$ S. Corezzi, D. Fioretto, and J. M. Kenny, Phys. Rev. Lett. 94, 065702 (2005).

${ }^{7}$ G. Adam and J. H. Gibbs, J. Chem. Phys. 43, 139 (1965).

${ }^{8}$ M. H. Cohen and D. Turnbull, J. Chem. Phys. 31, 1164 (1959).

${ }^{9}$ F. Spaepen, Acta Metall. 25, 407 (1977).

${ }^{10}$ A. S. Argon, Acta Metall. 27, 47 (1979).

${ }^{11}$ A. S. Argon and L. T. Shi, Acta Metall. 31, 499 (1983).

${ }^{12}$ C. A. Schuh and A. C. Lund, Nat. Mater. 2, 449 (2003).

${ }^{13}$ L. O. Eastgate, J. S. Langer, and L. Pechenik, Phys. Rev. Lett. 90,
045506 (2003).

${ }^{14}$ M. L. Falk, J. S. Langer, and L. Pechenik, Phys. Rev. E 70, 011507 (2004).

${ }^{15}$ J. S. Langer, Phys. Rev. E 64, 011504 (2001).

${ }^{16}$ M. L. Falk and J. S. Langer, Phys. Rev. E 57, 7192 (1998).

${ }^{17}$ P. G. Debenedetti and F. H. Stillinger, Nature (London) 410, 259 (2001).

${ }^{18}$ W. L. Johnson and K. Samwer, Phys. Rev. Lett. 95, 195501 (2005).

${ }^{19}$ M. S. Daw and M. I. Baskes, Phys. Rev. B 29, 6443 (1984).

${ }^{20}$ M. J. Sabochick and N. Q. Lam, Phys. Rev. B 43, 5243 (1991).

${ }^{21}$ S. G. Mayr, Y. Ashkenazy, K. Albe, and R. S. Averback, Phys. Rev. Lett. 90, 055505 (2003).

${ }^{22}$ S. G. Mayr, Phys. Rev. B 71, 144109 (2005).

${ }^{23}$ S. Vauth and S. G. Mayr, Appl. Phys. Lett. 86, 061913 (2005).

${ }^{24}$ H. J. C. Berendsen, J. P. M. Postma, W. F. van Gunsteren, A. DiNola, and J. R. Haak, J. Chem. Phys. 81, 3684 (1984).

${ }^{25}$ H. R. Schober, J. Non-Cryst. Solids 307-310, 40 (2002).

${ }^{26}$ C. Oligschleger and H. R. Schober, Phys. Rev. B 59, 811 (1999). 